\title{
An intronic variable number of tandem repeat polymorphisms of the cold-induced autoinflammatory syndrome 1 (CIAS1) gene modifies gene expression and is associated with essential hypertension
}

\author{
Toshinori Omi ${ }^{1}$, Maki Kumada ${ }^{1}$, Toyomi Kamesaki ${ }^{1}$, Hiroshi Okuda ${ }^{1}$, \\ Lkhagvasuren Munkhtulga $^{1}$, Yoshiko Yanagisawa ${ }^{1}$, Nanami Utsumi ${ }^{1}$, Takaya Gotoh ${ }^{1}$, \\ Akira Hata $^{2}$, Masayoshi Soma ${ }^{3}$, Satoshi Umemura ${ }^{4}$, Toshio Ogihara ${ }^{5}$, Norio Takahashi ${ }^{6}$, \\ Yasuharu Tabara ${ }^{7}$, Kazuyuki Shimada ${ }^{8}$, Hiroyuki Mano ${ }^{9}$, Eiji Kajii ${ }^{1}$, Tetsuro Miki ${ }^{7}$ \\ and Sadahiko Iwamoto ${ }^{* 1}$
}

\footnotetext{
${ }^{1}$ Division of Human Genetics, Center for Community Medicine, Jichi Medical School, Tochigi, Japan; ${ }^{2}$ Department of Public Health, Graduate School of Medicine, Chiba University, Chiba, Japan; ${ }^{3}$ Department of Nephrology and Endocrinology, Advanced Medical Research Center, Nihon University School of Medicine, Tokyo, Japan; ${ }^{4}$ Second Department of Internal Medicine, Yokohama City University Medical School, Kanagawa, Japan; ${ }^{5}$ Department of Genetics, Radiation Effects Research Foundation, Hiroshima, Japan; ${ }^{6}$ Department of Geriatric Medicine, Osaka University Graduate School of Medicine, Osaka, Japan; ${ }^{7}$ Department of Geriatric Medicine, Ehime University School of Medicine, Ehime, Japan; ${ }^{8}$ Division of Cardiovascular Medicine, Department of Medicine, Jichi Medical School, Tochigi, Japan; ${ }^{9}$ Division of Functional Genomics, Jichi Medical School, Kawachigun, Tochigi, Japan
}

Cold-induced autoinflammatory syndrome 1 (CIAS1) gene is a member of the NALP subfamily of the CATERPILLER protein family that is expressed predominantly in peripheral blood leukocytes, which is to regulate apoptosis or inflammation through the activation of NF- $\kappa$ B and caspase. Recent genetic analyses suggested an association between inflammation and oxidative stress-related genes in the development of hypertension. This is the first genetic study indicating an association between the CIAS1 gene and susceptibility to essential hypertension (EH). The frequency of subject with the homozygote of 12 repeat allele was significantly higher in patients with hypertension compared with control subjects (987 cases, 924 controls $)(P=0.030$; odds ratio $=1.24)$ at a novel VNTR polymorphism of CIAS1 intron 4 loci. We also found that the mean of systolic blood pressure of homozygotes of 12 repeat allele was $6.4 \mathrm{mmHg}$ higher than those of homozygotes of non-12 repeat allele in male random population $(P=0.009)$. The frequency of six SNPs spanning of the CIAS1 gene was not significantly between patients and controls. The real-time PCR analysis showed that among healthy young adults, 12-12 subjects expressed CIAS1 mRNA in peripheral leukocytes significantly more abundantly than homozygote of non-12 repeat alleles subjects $(P<0.05)$. Reporter gene assay of the CIAS1-VNTR in HL60 stimulated by lipopolysaccharides showed that the intronic sequence involving 12 repeat increased the expression of luciferase compared with 9, 7, and 6 repeats.

*Correspondence: Professor S Iwamoto, Division of Human Genetics, Center for Community, Medicine, Jichi Medical School, Minamikawachi-machi, Tochigi 329-0498, Japan. Tel: 8128544 2111; Fax: 8128544 4902;

E-mail: siwamoto@jichi.ac.jp

Received 6 October 2005; revised 29 May 2006; accepted 16 June 2006; published online 26 July 2006 
Thus, we propose here the CIAS1 is associated with EH through the dominant expression of transcripts, which may depend on the CIAS1-VNTR genotype.

European Journal of Human Genetics (2006) 14, 1295-1305. doi:10.1038/sj.ejhg.5201698; published online 26 July 2006

Keywords: association study; CIAS1; hypertension; VNTR polymorphis

Essential hypertension (EH [MIN1455]) is a complex disease that is thought to be influenced by polygenic and multiple environmental factors. Blood pressure is known to be a heritable trait, but the inherited genetic factors appear to have an influence on blood pressure and hypertension only $\sim 50 \%$ of the time. Recently susceptibility loci for $\mathrm{EH}$ and a healthy variation in blood pressure have been mapped on several human chromosomes by genome-wide scans. ${ }^{1-9}$ Although several dozen candidate genes for hypertension have been proposed, understanding the genetic influences on this disease is difficult because, when combining data from different studies, there is a lack of replication of an identified locus.

Recent biochemical and morphological studies suggest that remodeling of large and small arteries is involved in the development and associated complications of hypertension. ${ }^{10,11}$ In the past decade, inflammation and oxidative stress have emerged as major players in vascular remodeling of hypertension in addition to known mechanisms, such as salt sensitivity, insulin resistance, and an imbalance in the renin-angiotensin system. ${ }^{12-15}$ Polymorphonuclear leukocytes (PMNL) are the main producer of reactive oxygen species (ROS) and have been shown to be involved in hypertension in both human and animal models. There is evidence supporting the presence of activated PMNL in humans and rats with hypertension. In a hypertension model using Sabra hypertension-prone rats, superoxide release from PMNL and inflammation anteceded the onset of hypertension (brought on by salt loading). ${ }^{16,17}$ Results indicated that genetic factors contributed to the activation of PMNL and production of ROS. The p22phox is a major component of NADPH oxidase and is essential for $\mathrm{O}^{2-}$ generation. A polymorphism in the promoter region of the p22phox gene, $-930 \mathrm{~A} / \mathrm{G}$, has been shown to be associated with EH. ${ }^{18}$ The present study was designed to identify a novel hypertensive-susceptibility locus using a single-candidate-gene approach on a gene involved in the activation of PMNL.

The CATERPILLER family gene (CARD, transcription enhancer, R (purine)-binding, pyrin, lots of leucine protein family that is expressed predominantly in peripheral blood leukocytes ${ }^{19-25}$ in human. In addition, the rat orthologue of a member in CATERPILLER family gene, PYPAF5 (PYRINcontaining apoptotic protease-activating factor -1-like proteins 5), encodes angiotensin II/vasopressin dual receptor as a splicing isoform product ${ }^{26}$ and the variation cosegregate with salt-sensitive hypertension in Dahl salt- sensitive hypertension rat model. ${ }^{27}$ Then, we focused the relation between hypertension and the CATERPILLER family gene. The CIAS1 (MIN 606416; cold-induced autoinflammatory syndrome 1) gene mapped $1 \mathrm{q} 44^{19}$ encodes cryopyrin/NALP3/PYPAF1 (NACHT-LRR-PYD-containing protein-3/PYRIN-containing Apaf1-like protein-1) proteins, a member of the NALP subfamily of the CATERPILLER. The function of the proteins within the subfamily is thought to involve the regulation of apoptosis or inflammation through the activation of (nuclear factor) (NF)- $\kappa \mathrm{B}$ and caspase. Thus far, many missense mutations in the CIAS1 gene have been identified in hereditary systemic autoinflammatory disorders. ${ }^{28}$ However, an association between the CIAS1 gene and hypertension has not been reported. We used a case-control study using a singlecandidate-gene approach to determine whether the CIAS1 gene was associated with hypertension.

\footnotetext{
Methods

Subjects for genotyping

Population of panels 1 (100 cases and 109 controls) and 2 (987 cases and 924 controls) for case-control study were from the Tochigi prefecture, Ehime prefecture, Hiroshima prefecture, Osaka, or Kanagawa prefecture, Tokyo, Hokkaido in Japan (the study group from the Group of Millennium Genome Project for Hypertension). All subjects gave informed consent, and the study was approved by the ethics committee of Jichi Medical School. The hypertension group included patients who were diagnosed before 60 years of age; hypertension was defined as a blood pressure (systolic/diastolic) of $160 / 100 \mathrm{mmHg}$ or higher. We excluded hypertensive individuals who had diabetes, intrinsic renal disease, a self-reported history of secondary hypertension that was corroborated by a family physician, or coexisting illness. We aimed to recruit hypertensive individuals with a body mass index (BMI) less than 25 to exclude the patient including obesity. In addition, the hypertensive individuals were selected with at least one first-degree family history because we considered that the patients with family history much received the genetic influence of hypertension compare to the patients without family history. Control subjects after 50 years included those with a blood pressure of $120 / 80 \mathrm{mmHg}$ or lower and without hypertensive first-degree relatives. Blood pressure was measured with a mercury sphygmomanometer at least three times in a sitting position in clinic.
} 
Panel 3 from random population (268 men and 162 women) was collected from the individual who came a medical check up at the Health Examination Center in Jichi Medical School. Genotypes were compared with phenotypes; age, BMI, systolic blood pressure (SBP), diastolic blood pressure (DBP), total-cholesterol, highdensity lipoprotein (HDL), triglycerides, fasting blood sugar (FBS), and glycosylated hemoglobin (HbA1c).

\section{Genotyping of SNPs and a VNTR in the CIAS1 gene} Four single nucleotide polymorphisms (SNPs) (IMSJST084196, IMS-JST165517, IMS-JST165520, and IMSJST084195) selected from seven SNPs registered in the Japanese SNP database, ${ }^{20}$ a SNP (rs1539019) of NCBI and a SNP (HCV26052025) of commercial database (Celera Discovery System) were selected to cover the CIAS1 gene. Each SNP was genotyped by the Taq Man-PCR assay using the ABI PRISM 7900-HT according to the manufacture's instructions (Applied Biosystems, Foster, CA, USA). For an association study, we also used a novel variable number of tandem repeat polymorphisms (VNTR) polymorphism in intron 4 of the CIAS1 gene that was found through resequencing. To genotype the VNTR polymorphisms, standard PCR was performed in a final reaction volume of $25 \mu \mathrm{l}$ of $1 \times$ PCR buffer containing $20 \mathrm{ng}$ of the genomic DNA, $0.4 \mu \mathrm{M}$ of a set of primer (forward:5'-CTGACCTCC CAATGTGCCTT- $3^{\prime}$ and reverse:5'-CAGAGCTTCTTCA GATTGCA-3'), $0.2 \mathrm{mM}$ concentrations of each dNTP, and 2.5 U Taq DNA polymerase (Roche Dignostics, GmbH, Mannheim, Germany), using the following temperature profile: $95^{\circ} \mathrm{C}$ for $3 \mathrm{~min}$, followed by 35 cycles of $95^{\circ} \mathrm{C}$ for $1 \mathrm{~min}, 55^{\circ} \mathrm{C}$ for $1 \mathrm{~min}, 72^{\circ} \mathrm{C}$ for $1 \mathrm{~min}$, and a final extension of $72^{\circ} \mathrm{C}$ for $7 \mathrm{~min}$. The PCR products were electrophoresed on a $3 \%$ agarose gel and stained with ethidium bromide.

\section{Association between VNTR polymorphisms and gene expression in vivo}

Total RNA from leukocytes was isolated from the blood of 46 healthy young adults with different genotypes by the Aquapure RNA blood kit (Bio-Rad, CA, USA). The 46 RNA consisted of 21 homozyzotes of major repeats 12,16 heterozygozes of major and minor repeats $(9,7$, and 6 repeats), and nine homozygtes of minor repeats. We further analyzed the expression of CIAS1 in their primary monocytes after lipopolysaccharide (LPS) stimulation. Monocytes were isolated from peripheral leukocytes by standard Ficoll centrifugation procedure followed by adhesion on plastic dishes coated by auto-serum. The attached cells were stimulated with $200 \mathrm{ng} / \mathrm{ml}$ LPS (LPS 026:B6; Sigma-Aldrich, St Louis, MO) for 30 or $60 \mathrm{~min}$ at $37^{\circ} \mathrm{C}$ and lysed in Trizol reagent (Life Technologies). For real-time PCR, approximately $100 \mathrm{ng}$ total RNA from each cell was first reverse transcribed (final volume of $12.5 \mu \mathrm{l}$ ) into cDNA using the SuperScript II (Life Technologies, Gaithersburg, MD) according to the manufacture's proto- col. RT product $(0.2 \mu \mathrm{l})$ was used for quantification of gene expression by TaqMan real-time RT-PCR (Applied Biosystems, Forster City, CA, USA) in a reaction volume of $5 \mu \mathrm{l}$. The cumulative fluorescence for each amplicon was normalized to that seen with VIC-labeled GAPDH (Applied Biosystems, Foster, CA, USA) amplification. A FAM-labeled Hs00918086 probe (Applied Biosystems, Foster, CA, USA) was used for CIAS1 mRNA amplification.

\section{In vitro expression study by reporter gene assay of individual VNTR polymorphisms in HL60}

We constructed expression vectors using the pGL3 basic (Promega, Madison, WI) and pCAGGS vector. The four alleles $(12,9,7$, and 6 repeats unit) of CIAS1 42bp-VNTR were individually amplified from DNA of the subjects encoding the alleles using NheI- and NcoI-tailed primers (GATTACCATGGGCTAGCAATGTGCCTTGTG, and AGCACCCATGGTGGCGGCCACAGCGCCCCA), and the PCR products were digested by $\mathrm{NheI} / \mathrm{NcoI}$ and ligated into the pGL3 basic vector. After verification of the insert VNTR fragment by sequencing, VNTR-luciferase DNA fragment was extracted from the pGL3 vector by Nhel/ $X b a \mathrm{I}$ digestion and inserted in the pCAGGS vector at the $X b a \mathrm{I}$ site to mimic the splicing acceptor position. Ten micrograms of the constructs were transfected into $5 \times 10^{6}$ HL60 cells with $0.2 \mu \mathrm{g}$ of pRL vector (renilla luciferase, Promega) by electroporation procedure using GenePulser II (Bio-Rad Laboratories, Hercules, CA, USA). At $24 \mathrm{~h}$ after transfection, the relative light units (firefly/renilla light units) were measured by a dual luciferase reporter assay system (Promega) in a TD-20/20 luminometer (Turner Designs, Sunnyvale, CA, USA). For all transfection assays, at least three independent experiments were performed.

\section{Statistical analysis}

Values are expressed as mean \pm standard error of mean (SEM). Values of $P<0.05$ were considered statistically significant. Data were analyzed using a contingency table analysis and the Student's $t$-test. Hardy-Weinberg equilibrium was calculated by the $\chi^{2}$ test. Statistical analysis included a comparison of the proportions of the prevalence of the allele in cases and controls. Odds ratios (ORs) were generated from two-by-two tables, and statistical significance was assessed using the Fisher's extract. $\mathrm{D}^{\prime}$ and $r^{2}$ values were analyzed to evaluate a linkage disequilibrium (LD). Statistical analysis was performed using the SNPAlyze (Dynacom, Mobara, Japan) and SPSS statistical package (SPSS Japan, Tokyo).

\section{Results \\ Identification and characterization of a novel VNTR in the CIAS1 locus \\ A novel VNTR polymorphism was found in the process of CIAS1 resequencing, which was identified near the $3^{\prime}$ end}




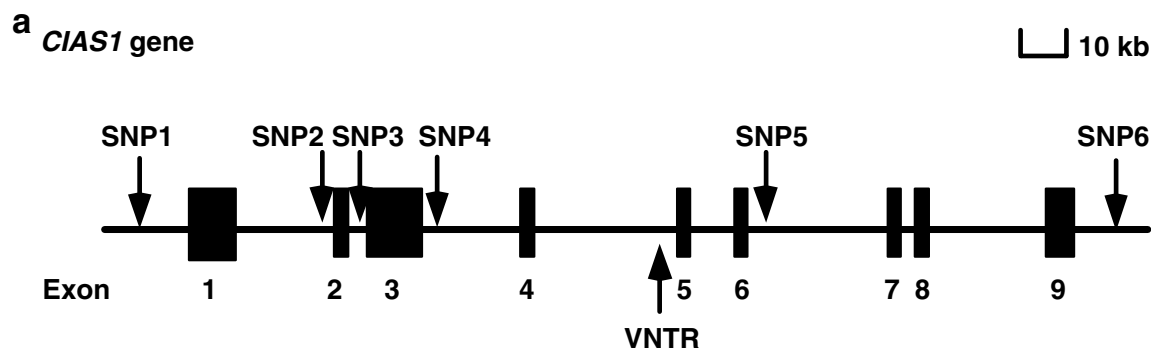

b

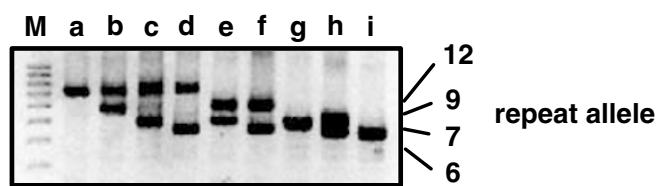

C

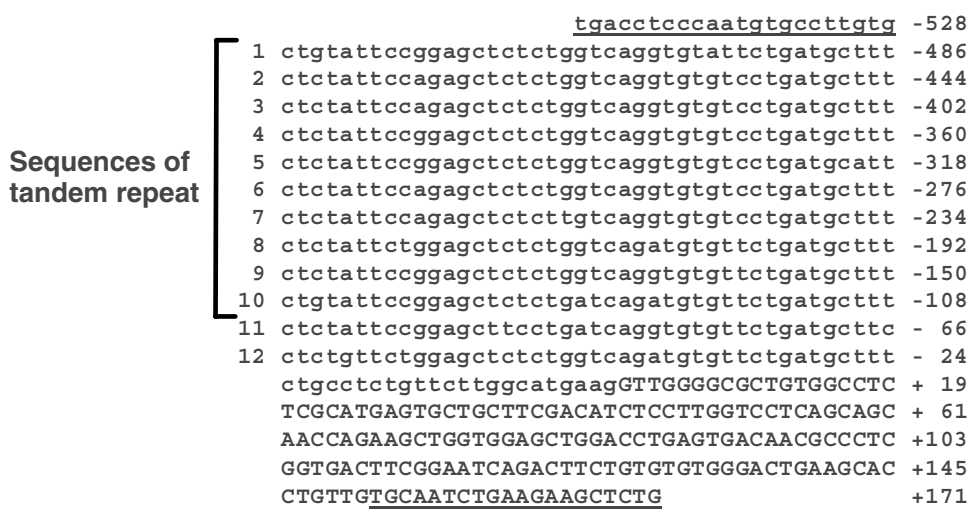

Figure 1 (a) Schema of CIAS1 gene with position of six SNPs and an identified VNTR polymorphisms for the genetic association study. Four SNPs were selected from the IMS-JST (Institute of Medical Science-Japan Science and Technology Agency) Japanese SNP database (IMS-JST): SNP1 (IMSJST084196), SNP2 (IMS-JST165517), SNP3 (IMS-JST165520), and SNP4 (IMS-JST084195). SNP5 (rs1539019) and SNP6 (hCV26052025) were selected from a NCBI database and a commercial database (Celera Discovery System), respectively. (b) Nine genotypes of a novel VNTR polymorphism in CIAS1, a:12-12, b:12-9, c :12-7, d:12-6, e:9-7, f:9-6, g:7-7, h:7-6, and i:6-6 were detected in this study as shown in the agarose gel electrophoresis. $\mathrm{M}$ is $100 \mathrm{bp}$ molecular ladder marker. (c) The nucleotide sequences of the 12 repeat allele of the CIAS1-42 bp VNTR. The tandem repeat number in each allele is 12 (GenBank Ac. No AB120959), 9 (GenBank Ac. No AB120960), 7 (GenBank Ac. No AB120961), and 6 (GenBank Ac. No AB120962).

of CIAS1 intron $4,-527$ to $-24 \mathrm{bp}$ from the splicing acceptor site of CIAS1 exon 5 (Figure 1a). The PCR analysis identified nine genotypes derived from four alleles (Figure 1b). Each allele consisted of the 720, 594, 510, and $468 \mathrm{bp}$ nucleotide sequences, respectively. The multiple alignment analysis between each allele showed the existence of the $42 \mathrm{bp}$ tandem repeat sequences in the CIAS1 gene (CIAS1 42 bp-VNTR). The nucleotide sequences of the 12 repeat allele was shown in Figure 1c. This tandem repeat polymorphism consisted of the 12, 9, 7, and 6 repeats units, respectively (GenBank accession number AB120959 to AB120962). The 12 repeat alleles' largest band was similar to the published sequence of the Homo sapiens chromosome 1 clone RP11-433K2 in GenBank (accession number AC104335). Estimation of the allele frequencies showed that the $12,9,7$, and 6 repeat units was 0.577 , $0.008,0.248$, and 0.167 in the CIAS1 $42 \mathrm{bp}$-VNTR locus $(n=507)$. The percentage of the all genotypes was $32.9 \%$ $(12-12), 1 \%(12-9), 30 \%$ (12-7), 18.5\% (12-6), 0\% (9-9),
$0.2 \%$ (9-7), 0.4\% (9-6), 6.5\% (7-7), 6.5\% (7-6), and 4\% (6-6), respectively. The observed genotype frequencies of all polymorphisms did not deviate from the predictions of the Hardy-Weinberg equation.

To assess whether the CIAS1 locus is associated with hypertension, the CIAS1 $42 \mathrm{bp}$-VNTR was genotyped for 109 control subjects and 100 patients with $\mathrm{EH}$ in panel 1. The frequency of subject with the homozygote of 12 repeat allele was significantly higher in patients with hypertension compared with control subjects $(P=0.04$; odds ratio $=1.84$ ) (Table 1$)$. The allele frequency of 12 repeat units, major allele, also tended to increase in patients compared with controls.

\section{Single-locus analyses and case-control haplotype analyses of the CIAS1 gene}

To reveal whether the CIAS1 $42 \mathrm{bp}$-VNTR is in tight linkage with other polymorphisms, six SNPs were chosen from the CIAS1 gene using a public database (JSNP, http:// 
Table 1 Genotyping data and association test of DNA polymorphisms on the CIAS1 in panel 1(100 cases and 109 controls)

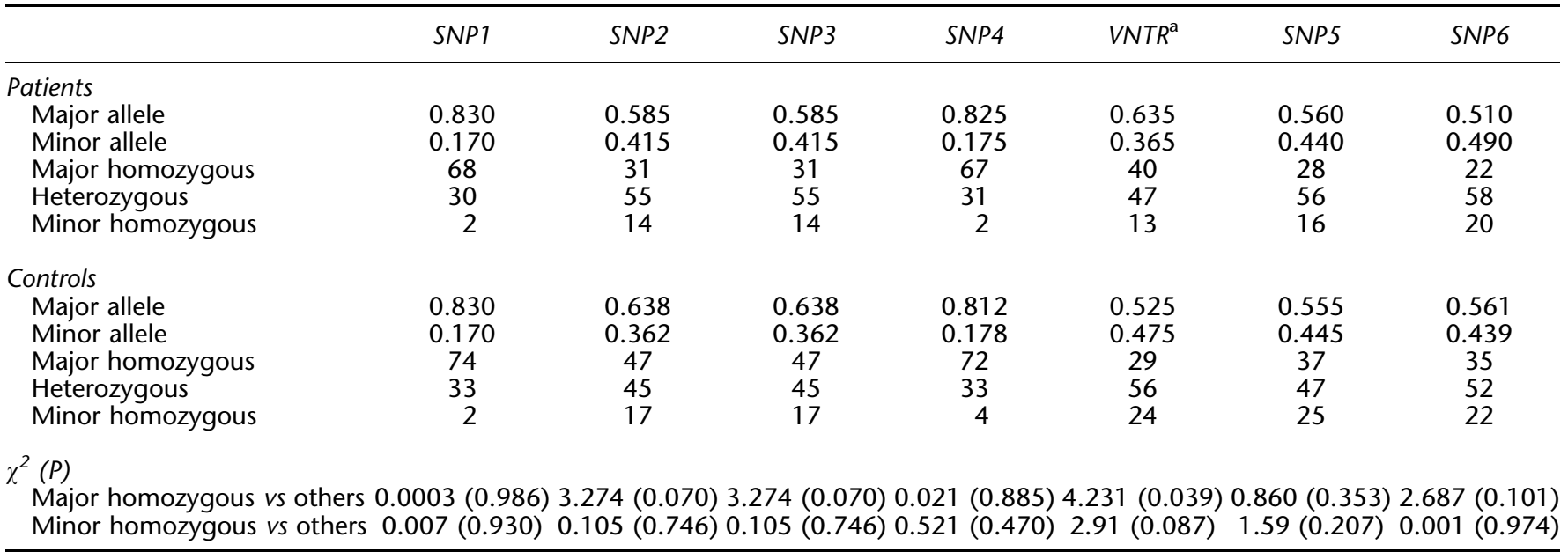

${ }^{a}$ Major allele is 12 repeat and minor alleles are 9,7 and 6 repeat.

Table 2 Frequency of the major haploype constructed from SNPs 1 to 6 between 109 controls and 100 cases

\begin{tabular}{lccc}
\hline Haplotypes & Controls (\%) & Case (\%) & $P$ \\
\hline CTACGT & 32.9 & 31.1 & NS \\
CTACTC & 16.2 & 13.3 & NS \\
CCGCTC & 13.1 & 17.5 & NS \\
CTACTT & 12.9 & 10.1 & NS \\
ACGTTC & 9.6 & 11.2 & NS \\
ACGTGT & 5.6 & 2.7 & NS \\
CCGCGT & 2.9 & 4.7 & NS \\
CCGTTC & 1.8 & 2.0 & NS \\
CCGCGC & 1.3 & 5.9 & NS \\
\hline
\end{tabular}

NS: not significance.

www.snp.ims.u-tokyo.ac.jp, Figure $1 \mathrm{a})^{29}$ for genotyping in panel 1 subjects. In the VNTR loci, the subjects were categorized into three genotypes: major allele homozygote (12-12), heterozygote with major and minor alleles (12-9, 12-7, and 12-6), and minor allele homozygote (9-9, 9-7, $9-6,7-6$, and 7-7), respectively. As a results, no significant association was shown between cases and controls in the six SNPs of the CIAS1 gene (Table 1).

Table 2 showed that the difference of haplotype frequency between the cases and controls. Our set of SNPs generated 10 common haplotypes (frequency $>0.01$ ) constructed from SNPs 1 to 6 , and there were no differences in haplotype frequencies between cases $(n=100)$ and controls $(n=109)$. We also analyzed the pairwise linkage disequilibrium (LD) by calculating $D^{\prime}$ and $r^{2}$ using genotype results (Table 3); $\left|D^{\prime}\right|>0.5$ and $r^{2}>0.5$ were regarded as tight linkage. Among the six SNPs, SNPs 1 ( $5^{\prime}$ franking region) to 4 (intron 4) tended to be in tight linkage but the LD was not distributed across the CIAS1 gene. The LD and casecontrol haplotype analysis of the CIAS1 gene suggest that
Table 3 Parwise LD in CIAS1 gene, evaluated by $D^{\prime}$ and $r^{2}$ from panel 1

\begin{tabular}{llllllll}
\hline & SNP1 & SNP2 & SNP3 & SNP4 & VNTR & SNP5 & SNP6 \\
\hline SNP1 & & & & & & \\
SNP2 $\left|D^{\prime}\right|$ & 0.940 & & & & & \\
$r^{2}$ & 0.286 & & & & & \\
SNP3 $\left|D^{\prime}\right|$ & 0.940 & 1.000 & & & & \\
$r^{2}$ & 0.286 & 1.000 & & & & \\
SNP4 $\left|D^{\prime}\right|$ & 0.912 & 1.000 & 1.000 & & & \\
$r^{2}$ & 0.765 & 0.351 & 0.351 & & & \\
VNTR $\left|D^{\prime}\right|$ & 0.715 & 0.145 & 0.145 & 0.841 & & \\
$r^{2}$ & 0.142 & 0.082 & 0.082 & 0.214 & & \\
SNP5 $\left|D^{\prime}\right|$ & 0.294 & 0.405 & 0.405 & 0.348 & 0.185 & \\
$r^{2}$ & 0.014 & 0.082 & 0.082 & 0.021 & 0.020 & & \\
SNP6 $\left|D^{\prime}\right|$ & 0.475 & 0.534 & 0.534 & 0.501 & 0.094 & 0.810 & \\
$r^{2}$ & 0.053 & 0.209 & 0.209 & 0.066 & 0.007 & 0.452 & \\
\hline
\end{tabular}

the VNTR locus was possibly independent from other SNPs and a functional polymorphism associated with hypertension.

\section{Case-control study of the CIAS1 $42 \mathrm{bp}$-VNTR for hypertension in a large sample}

To confirm the possibility that the homozygote of 12 repeat allele is a primary variant that determines susceptibility to hypertension, genotyping of CIAS1 $42 \mathrm{bp}$-VNTR was performed in panel 2 (987 cases and 924 controls). The results confirmed the statistical significance between the CIAS1 $42 \mathrm{bp}-\mathrm{VNTR}$ and EH (Table 4). The significant difference was $P=0.030$ (Odds 1.24) in the comparison of $12-12$ vs $12-\mathrm{X}+\mathrm{X}-\mathrm{X}$ and $P=0.006$ (Odds 1.39 ) in $12-12$. $+12-\mathrm{X} v s \mathrm{X}-\mathrm{X}$. These results led us the homozygote of 12 repeat alleles was an association with risk of $\mathrm{EH}$. The frequency of the 12-12 subjects was 0.348 and 0.301 in panel 2 . The most significant association was shown in the 
Table 4 Association of the CIAS1-VNTR genotype with essential hypertension in panel 2

\begin{tabular}{|c|c|c|c|c|c|}
\hline Panel 2 & Controls & Patients & $\chi^{2}$ & $P$ & Odds \\
\hline \multicolumn{6}{|l|}{ Genotype distribution } \\
\hline $12-12$ & $278(30.1 \%)$ & $343(34.7 \%)$ & & & \\
\hline $12-X$ & $466(50.4 \%)$ & $498(50.5 \%)$ & & & \\
\hline$X-X$ & $180(19.5 \%)$ & $146(14.8 \%)$ & & & \\
\hline \multicolumn{6}{|l|}{ Allele frequency } \\
\hline 12 & 0.553 & 0.600 & & & \\
\hline$x$ & 0.447 & 0.400 & & & \\
\hline \multicolumn{6}{|l|}{ Genotype comparison } \\
\hline $12-12$ vs $12-\mathrm{X}+\mathrm{X}-\mathrm{X}$ & & & 4.735 & 0.030 & 1.24 \\
\hline $12-12+12-\mathrm{X}$ vs $\mathrm{X}-\mathrm{X}$ & & & 7.413 & 0.006 & 1.39 \\
\hline
\end{tabular}

Table 5 Clinical characterization of random population of panel 3 to CIAS1 42 bp-polymorphism

\begin{tabular}{|c|c|c|c|c|}
\hline Panel 3 (Men and Women) & $12-12$ & $\begin{array}{c}\text { Genotype } \\
12-X\end{array}$ & $X-X$ & $P$ \\
\hline$N(\%)$ & $138(32.1 \%)$ & $218(50.7 \%)$ & $74(17.2 \%)$ & NS \\
\hline Age & $51.4 \pm 8.7$ & $52.1 \pm 9.5$ & $53.5 \pm 8.6$ & NS \\
\hline Height (cm) & $163.0 \pm 6.8$ & $163.2 \pm 8.8$ & $163.3 \pm 8.5$ & NS \\
\hline Weight (kg) & $63.7 \pm 9.1$ & $63.1 \pm 11.2$ & $62.5 \pm 10.2$ & NS \\
\hline $\operatorname{BMI}\left(\mathrm{kg} / \mathrm{m}^{2}\right)$ & $24.0 \pm 2.7$ & $23.8 \pm 3.4$ & $23.3 \pm 2.6$ & NS \\
\hline SBP $(\mathrm{mmHg})$ & $127.7 \pm 14.2$ & $124.3 \pm 16.4$ & $122.4 \pm 13.3$ & $0.018^{\star}$ \\
\hline $\mathrm{DBP}(\mathrm{mmHg})$ & $78.6 \pm 9.7$ & $77.6 \pm 11.1$ & $76.3 \pm 9.8$ & NS \\
\hline Total-cholesterol (mg/dl) & $205.2 \pm 34.4$ & $205.6 \pm 35.8$ & $198.6 \pm 32.3$ & NS \\
\hline $\mathrm{HDL}(\mathrm{mg} / \mathrm{dl})$ & $61.8 \pm 13.9$ & $60.9 \pm 18.2$ & $61.9 \pm 14.3$ & NS \\
\hline Triglyceride $(\mathrm{mg} / \mathrm{dl})$ & $109.3 \pm 53.8$ & $103.0 \pm 51.7$ & $96.2 \pm 43.1$ & NS \\
\hline Fasting blood glucose $(\mathrm{mg} / \mathrm{dl})$ & $100.5 \pm 8.7$ & $98.9 \pm 20.2$ & $101.4 \pm 15.8$ & NS \\
\hline $\mathrm{HbA1c}(\%)$ & $5.0 \pm 0.3$ & $5.1 \pm 0.4$ & $5.1 \pm 0.4$ & NS \\
\hline
\end{tabular}

NS; not significant.

T-Cho, total cholesterol; HDL-Cho, HDL cholesterol; TG, triglyceride, FBS, Fasting blood sugar; and HbA1c, glycohemoglobin, A-1-C. *12-12 vs $12-\mathrm{X}+\mathrm{X}-\mathrm{X}$.

comparison of $12-12$ s $\mathrm{X}-\mathrm{X}\left(\chi^{2}=13.048, P=0.0003\right.$, odds ratio $=1.60) \quad($ data not shown). These genetic results indicate that the CIAS1 locus contributes to the susceptibility to $\mathrm{EH}$.

\section{Association between CIAS1 42 bp-VNTR genotype and random population in blood pressure}

We further examined the association between the CIAS1 gene and healthy variations in blood pressure. Panel 3 from random population (268 men and 162 women) was collected at the Health Examination Center in Jichi Medical School, which was independent of panels 1 and 2. Genotypes were compared with phenotypes; age, BMI, SBP, DBP, total-cholesterol, HDL, triglycerides, FBS, and HbA1c. Table 5 showed the genotype effect in three groups of $12-12,12-\mathrm{X}$, and $\mathrm{X}-\mathrm{X}$ to clinical characteristics in CIAS1 gene. We found the significant difference of the SBP in three genotype groups by one-way ANOVA study in panel $3(P=0.018)$. The association between the genotype and the SBP was revealed in men $(P=0.028$, Table 6) but not in women (Table 7). The mean SBP in
$12-12$ subjects $(n=86)$ was $6.4 \mathrm{mmHg}$ higher than those of X-X subjects $(n=44)$ in men. The CIAS1 $42 \mathrm{bp}-\mathrm{VNTR}$ genotype was not associated with BMI, DBP, total-cholesterol, HDL, triglycerides, fasting blood glucose, and HbA1c levels. To exclude the influence of age on BP or population stratification among generation, age was put into covariate and analysis of covariance test (ANCOVA) was carried out. After this correction significant difference of SBP was observed again among three genotypes in men $(P=0.028)$, and the significance became distinct $(P=0.011)$, when over 55 years old men were analyzed. In 162 women, we did not find a significant difference of blood pressure among the three groups in ANOVA or ANCOVA study even after age correction, but the mean SBP of 12-12 subjects was slightly higher than that of other groups (Table 7 ).

Genotype and the expression level of CIAS1 transcript The transcript from CIAS1 was predominantly expressed in peripheral blood leukocytes compared with total RNA from bone marrow, brain, fetal liver heart, kidney, lung, 
Table 6 Clinical characterization of random population of panel 3 to CIAS1 42 bp-polymorphism in Men

\begin{tabular}{|c|c|c|c|c|}
\hline Panel 3 (Men) & $12-12$ & $\begin{array}{c}\text { Genotype } \\
12-X\end{array}$ & $X-X$ & $P$ \\
\hline$N(\%)$ & $86(32.1 \%)$ & $138(51.5 \%)$ & $44(16.4 \%)$ & NS \\
\hline Age & $53.5 \pm 8.5$ & $52.6 \pm 9.3$ & $54.1 \pm 7.2$ & NS \\
\hline Height $(\mathrm{cm})$ & $167.0 \pm 6.8$ & $167.9 .1 \pm 6.1$ & $168.7 \pm 5.1$ & NS \\
\hline Weight (kg) & $67.7 \pm 9.3$ & $68.4 \pm 8.6$ & $67.2 \pm 8.6$ & NS \\
\hline $\mathrm{BMI}\left(\mathrm{kg} / \mathrm{m}^{2}\right)$ & $24.2 \pm 2.7$ & $24.2 \pm 2.3$ & $23.6 \pm 2.7$ & NS \\
\hline SBP $(\mathrm{mmHg})$ & $131.7 \pm 14.2$ & $126.8 \pm 16.0$ & $125.4 \pm 13.6$ & 0.028 \\
\hline $\mathrm{DBP}(\mathrm{mmHg})$ & $80.6 \pm 9.7$ & $79.7 \pm 11.1$ & $79.0 \pm 9.6$ & NS \\
\hline Total-cholesterol $(\mathrm{mg} / \mathrm{dl})$ & $207.3 \pm 34.4$ & $206.4 \pm 35.1$ & $197.9 \pm 32.9$ & NS \\
\hline $\mathrm{HDL}(\mathrm{mg} / \mathrm{dl})$ & $58.7 \pm 13.9$ & $55.8 \pm 13.3$ & $56.8 \pm 13.9$ & NS \\
\hline Triglyceride (mg/dl) & $127.6 \pm 53.8$ & $119.1 \pm 53.7$ & $112.2 \pm 44.9$ & NS \\
\hline Fasting blood glucose $(\mathrm{mg} / \mathrm{dl})$ & $104.2 \pm 8.7$ & $103.0 \pm 11.5$ & $102.8 \pm 11.7$ & NS \\
\hline $\mathrm{HbA1c}(\%)$ & $5.0 \pm 0.3$ & $5.1 \pm 0.4$ & $5.0 \pm 0.6$ & NS \\
\hline
\end{tabular}

NS; not significant.

T-Cho, total cholesterol; HDL-Cho, HDL cholesterol; TG, triglyceride, FBS, Fasting blood sugar; and HbA1C, glycohemoglobin, A-1-C.

Table 7 Clinical characterization of random population of panel 3 to CIAS1 42 bp-polymorphism in Women

\begin{tabular}{|c|c|c|c|c|}
\hline Panel 3 (Women) & $12-12$ & $\begin{array}{c}\text { Genotype } \\
12-X\end{array}$ & $X-X$ & $P$ \\
\hline$N(\%)$ & $52(32.1 \%)$ & $80(49.4 \%)$ & $30(18.5 \%)$ & NS \\
\hline Height (cm) & $156.3 \pm 5.115$ & $5.1 \pm 6.7$ & $155 \pm 5.7$ & NS \\
\hline Weight (kg) & $57.1 \pm 7.8$ & $54.0 \pm 9.1$ & $55.5 \pm 8.1$ & NS \\
\hline $\mathrm{BMI}\left(\mathrm{kg} / \mathrm{m}^{2}\right)$ & $23.5 \pm 3.5$ & $23.1 \pm 4.7$ & $22.9 \pm 2.4$ & NS \\
\hline SBP $(\mathrm{mmHg})$ & $121.0 \pm 13.5$ & $120.1 \pm 16.4$ & $118.1 \pm 11.5$ & NS \\
\hline $\mathrm{HDL}(\mathrm{mg} / \mathrm{dl})$ & $67.0 \pm 15.6$ & $67.9 \pm 15.6$ & $69.2 \pm 11.3$ & NS \\
\hline Triglyceride (mg/dl) & $79.2 \pm 49.0$ & $75.6 \pm 33.5$ & $72.3 \pm 26.5$ & NS \\
\hline Fasting blood glucose (mg/dl) & $94.3 \pm 10.3$ & $92.0 \pm 28.5$ & $99.4 \pm 20.2$ & NS \\
\hline $\mathrm{HbA1c}(\%)$ & $5.0 \pm 0.4$ & $5.1 \pm 0.3$ & $5.1 \pm 0.4$ & NS \\
\hline
\end{tabular}

NS; not significant.

T-Cho, total cholesterol; HDL-Cho, HDL cholesterol; TG, triglyceride, FBS, Fasting blood sugar; and HbA1c, glycohemoglobin, A-1-C.

placenta, prostate, salivary gland, skeletal muscle, spleen, testis, thyroid gland, trachea, uterus, colon, small intestine, spinal cord, and stomach packaged in the master panel (BD Biosciences, CA, USA) by RT-PCR analysis (data not shown). We next tested the genotype effect of CIAS1 $42 \mathrm{bp}-$ VNTR locus to the expression level of CIAS1 transcript using the total leukocyte RNA extracted from blood samples in healthy young volunteers with different genotypes. Real-time PCR analysis showed that the expression level of the CIAS1 transcript was different in subjects with 12-12 $(n=21), 12-\mathrm{X}(n=16)$, and X-X $(n=9)$ VNTR genotypes (Figure 2 ). The CIAS1 transcript was expressed significantly more in the $12-12$ subjects compared with subjects with the X-X genotype. Real-time PCR analysis showed that the expression level of the CIAS1 transcript showed significant difference in subjects with among three genotype groups $(P=0.009)$ by One-way ANOVA tests.
(Figure 2). Among the three genotype groups, the CIAS1 transcript from 12-12 subjects was much expressed than that of $12-\mathrm{X}$ and $\mathrm{X}-\mathrm{X}$ subjects.

\section{Expression of the transcript from CIAS1 locus in monocytes after LPS stimulation}

The CIAS1 expression level in total leukocytes showed remarkably high SD in both $12-12$ and $12-\mathrm{X}$ subjects (Figure 2), which was estimated to be dependent on the activated state of the leukocytes. To rule-out the possibility that the deviations simply resulted from the condition of donors or preparation procedure of leukocytes, the transcript level was also analyzed in monocytes under stimulation of LPS. As shown in Figure 3, transcript level in monocytes before stimulation of 12-12 subjects was higher than other genotype groups. One-way ANOVA tests showed the expression level of CIAS1 mRNA on monocyte 


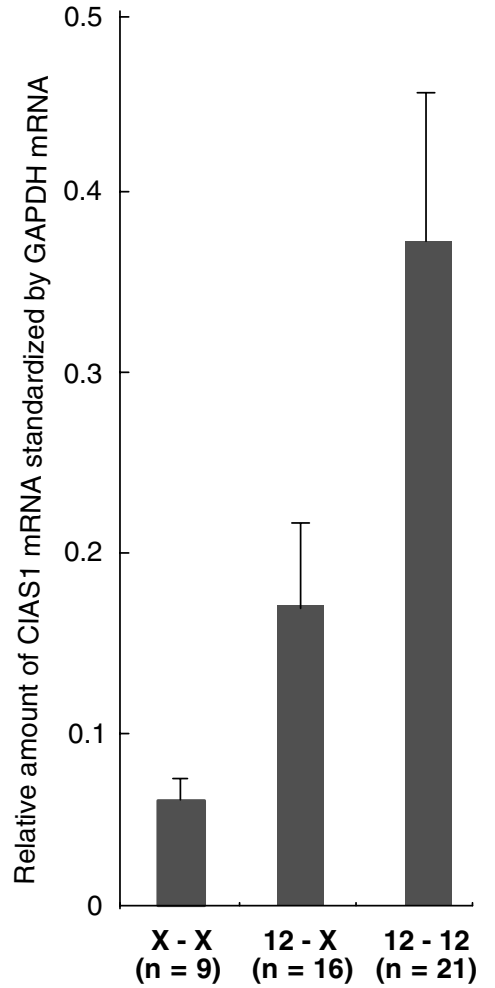

Figure 2 The CIAS1 mRNA levels in peripheral leukocytes among the three genotype groups of 12-12 (homozygote of 12 repeat alleles), $12-X$ (heterozygote of 12 and non- 12 repeat alleles), and $X-X$ (homozygote of non-12 repeat alleles) in CIAS1 $42 \mathrm{bp}$-VNTR. The CIAS1 expression was analyzed by real-time PCR. The relative amount of CIAS1 mRNA normalized by GAPDH is shown as fold volume. $n$ : number of subjects. One-way ANOVA tests showed that expression level of the CIAS1 transcript was significant difference in subjects among three genotype groups $(P=0.009)$.

was the significant difference on $30 \mathrm{~min}(P=0.01)$ and $60 \mathrm{~min}(P=0.0001)$ after LPS stimulation. These results indicated that the 12 repeat allele had an additive effect on the expression level of this gene.

\section{Functional assay of CIAS1 VNTR in HL 60}

To investigate whether CIAS1 $42 \mathrm{bp}$-VNTR directly affected gene expression, we next examined the reporter gene assay. We constructed the pCAGGS-based expression plasmid, containing the CIAS1 42 bp-VNTR alleles (12, 9, 7, and 6 repeat units) linked to the luciferase gene at the splicing acceptor site (Figure 4a). The pCAGGS promoter activity of the 12 VNTR repeat construct was higher than other repeat constructs before LPS stimulation in HL60 cells, although the difference was not significant (Figure $4 \mathrm{~b}$ ). The difference, however, became significant after 1 or $2 \mathrm{~h}$ of LPS stimulation. These results may confirmed the additive effect of 12 repeat allele on the expression level of CIAS1 gene (Figure 3) in the functional assay.

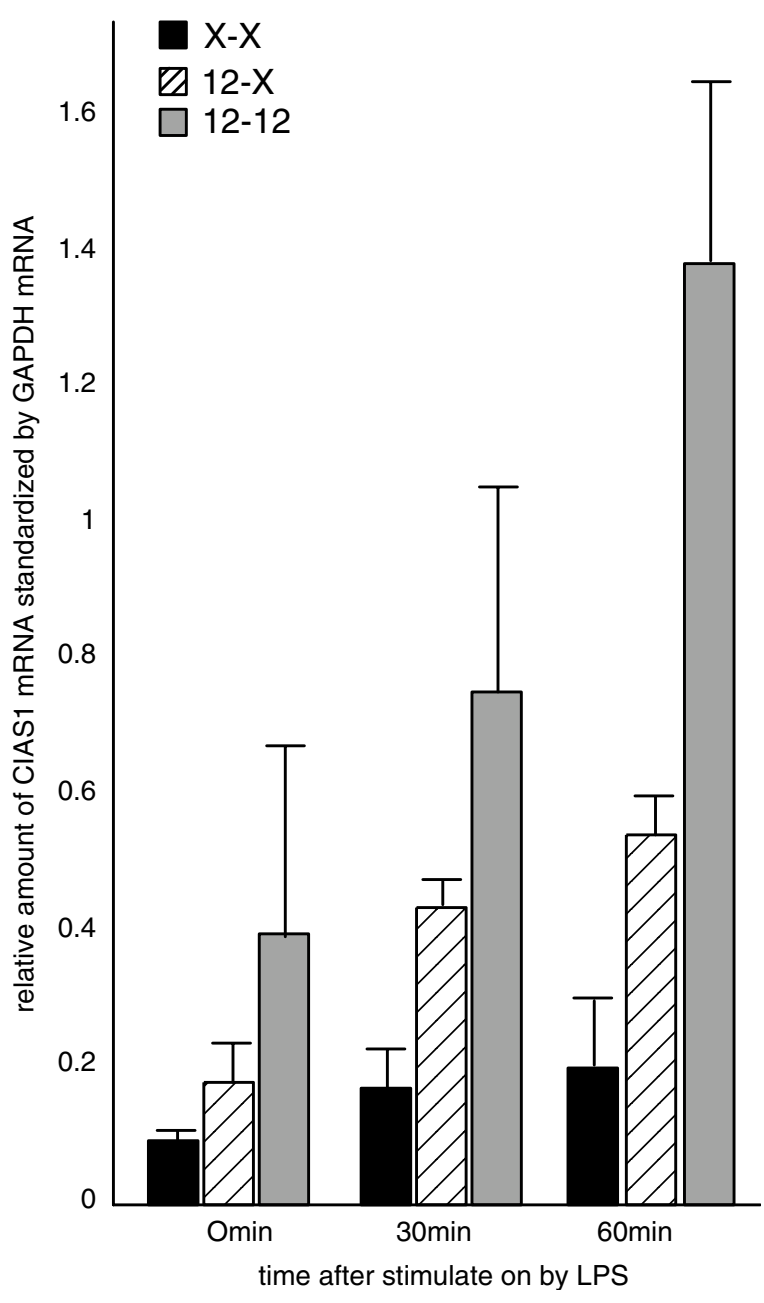

Figure 3 The effect of stimulation of LPS on CIAS1 mRNA levels in monocytes in different genotypes. Monocytes were isolated from peripheral leukocytes by Ficoll centrifugation followed by adhesion on a plastic dish. Cells were treated with $200 \mathrm{ng} / \mathrm{ml}$ LPS for 30 or $60 \mathrm{~min}$ and lysed. The relative amount of CIAS1 mRNA normalized by GAPDH is shown. Black bars show the transcripts with $X-X$ genotype (homozygote of non-12 repeat alleles), hatched bars indicate the transcripts with 12-X genotype (heterozygote of 12 repeat allele and non-12 alleles), and gray bars show the transcript with 12-12 genotype (homozygote of 12 repeat allele). One-way ANOVA tests showed the expression level of CIAS1 mRNA on monocyte was the significant difference on $30 \mathrm{~min}(P=0.01)$ and $60 \mathrm{~min}(P=0.0001)$ after LPS stimulation. Three individuals with each genotypes were tested.

\section{Discussion}

A single-candidate-gene approach for hypertension focusing on the CATERPILLER protein family has not been demonstrated. This is the first genetic study indicating an association between the CIAS1 gene and susceptibility to EH. Our study of 987 cases and 924 controls showed that subjects with 12-12 genotype of the CIAS1 $42 \mathrm{bp}$-VNTR experienced hypertension more frequently than controls, with a significant association $(P=0.006)$. Furthermore, the 
a
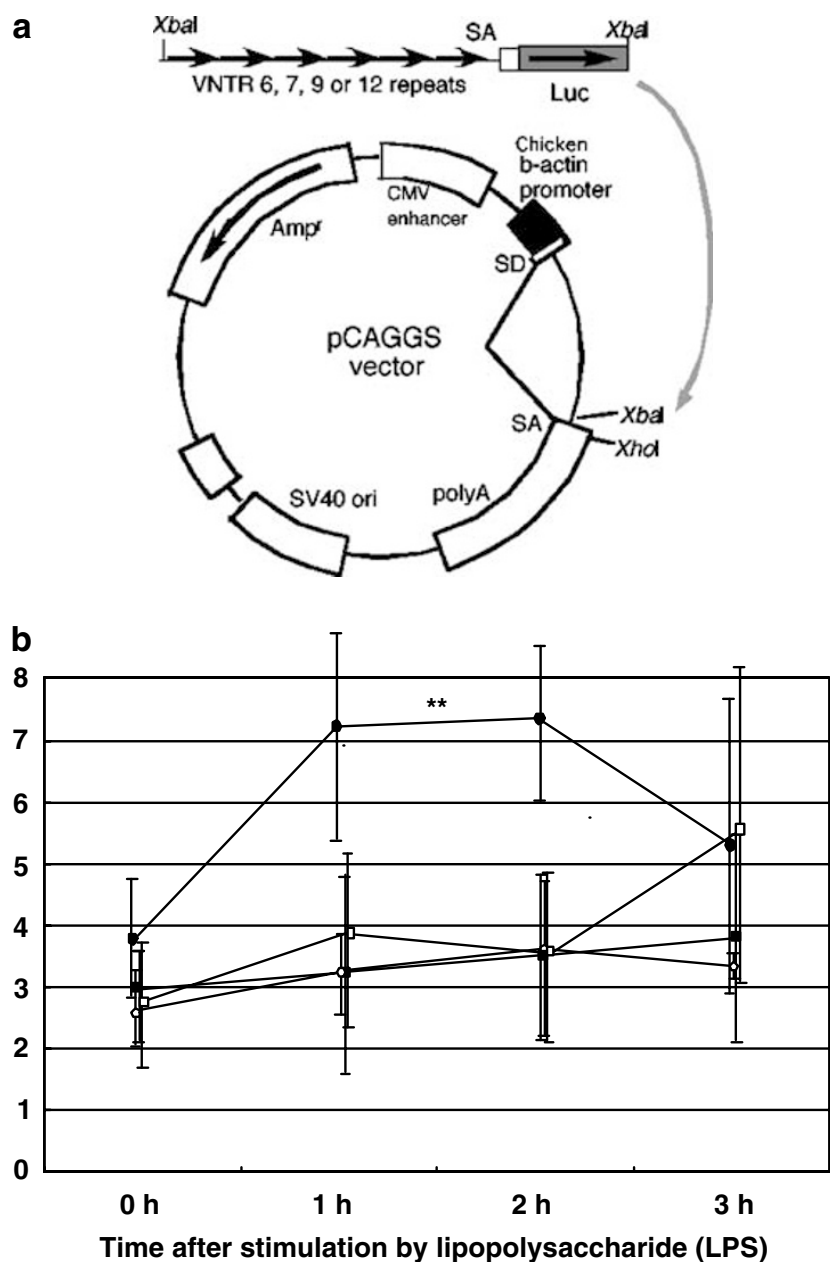

Figure 4 Functional assay of VNTR in HL60 cells. (a) The genomic region including the VNTR repeats, 12 (720 bp), 9 (594 bp), 7 $(510 \mathrm{bp})$, and 6 (468 bp), was inserted in pGL3 vector. The VNTRluciferase inserts were directionally subcloned in the PCAGGS vector. (b) HL60 cells were co-transfected with the pRL vector (renilla luciferase) by electroporation procedure. At $24 \mathrm{~h}$ after the transfection, HL60 cells were stimulated by LPS. The relative light units (firefly/ renilla light units) were measured by dual luciferase reporter assay system. PCAGGS-VNTR-12 (- -) vector PCAGGS-VNTR-9 (-O-), PCAGGS-VNTR-7 (-ם-), and PCAGGS-VNTR-6 (- $\square-)$. The relative luciferase activity with 12 repeat allele was significantly enhanced compared with the activity with 9,7 , and 6 repeat until more than $2 \mathrm{~h}$ after LPS.

comparison with genotype and clinical data in a random population showed that SBP was significantly higher in 12-12 subjects compared with $12-\mathrm{X}$ and $\mathrm{X}-\mathrm{X}$ subjects, although there was no difference in other variables. These results lead us to question whether the CIAS1 $42 \mathrm{bp}$-VNTR in intron is a true functional polymorphism in hypertension. We showed that the transcript level of CIAS1 in peripheral leukocytes and monocytes of healthy young adults was more abundant in 12-12 subjects than in those with the other genotypes. Furthermore, our reporter assay for CIAS1 $42 \mathrm{bp}$-VNTR showed that the 12 repeat units increased the expression of luciferase in the CIAS1 gene. These results may indicate that the CIAS1 $42 \mathrm{bp}-\mathrm{VNTR}$ directly affects gene expression. Thus, we believe that increased expression of CIAS1 in leukocytes dependent on the CIAS1 $42 \mathrm{bp}$-VNTR length may contribute to the development of $\mathrm{EH}$.

CIAS1 is a member of the recently discovered NALP/ PYPAF subfamily of the CATERPILLER protein family, thought to function in apoptotic and inflammatory signaling pathways. $^{27}$ CIAS1/NALP3/PYPAF1 protein is composed of three domains; the $\mathrm{N}$-terminal Pyrin domain, the central a specific nucleotide binding (NACHT) domain, and the C-terminal tandem copy of Leucin-rich repeats (LRRs). A large number of nucleotide substitutions have been found in the NACHT domain ${ }^{19}$ in patients with autosomal-dominant autoinflammatory disorders; MuckleWells syndrome, familial cold ulticaria, and chronic infantile neurologic cutaneous and articular syndrome. ${ }^{19,27}$ It has been proposed that the missense mutation in the NACHT domain weakens the self-regulation via its interaction with the LRRs and enables spontaneous activation by releasing NACHT domain from LRRs. The activated form recruits an apoptosis-associated speck-like protein containing a CARD (ASC) and Cardinal (TUCAN, CARD8, NDDP1). The caspase recruit domain (CARD) of ASC and Cardinal interact with that of procaspase-1. The resulting macrocomplex, called inflammasome, is available to convert pro-IL- $1 \beta$ into matured IL- $1 \beta$. Moreover, ASC and Cardinal in the inflammasome have been shown to be associated with IKK (I $\kappa$ B kinase) complex and activate NF- $\kappa \mathrm{B}$. The phenotypic heterogeneity (cold sensitivity, sensorineural hearing loss, severe neurologic involvement) has been observed in autoinflammatory syndromes. Although, the phenotypic heterogeneity has been mainly attributed to the mutation position in the NACHT domain, the amount of mutant CIAS1 mRNA might also contribute to the development of the syndrome.

The physiological function of the wild type CIAS1 is, however, still under investigation. Opposite results about $\mathrm{NF}-\kappa \mathrm{B}$ activation have been reported from overexpression analysis of CIAS 1 in culture cell lines. NF- $\kappa \mathrm{B}$ was activated by the overexpression of CIAS1 and ASC in HEK293 cells. ${ }^{21}$ The CIAS1 suppressed the activation in HeLa cells after TNF stimulation and suggested that a molecular balance of CIAS1 with ASC is critical for the inflammatory response. ${ }^{23,24}$ Recently Cias1-knockout mice are developed in two independent research groups. Mariathasan et al showed that Cryopyrin produced from CIAS1 activates the inflammasome in response to toxins and ATP. ${ }^{30}$ Kanneganti et al repots that bacteria RNA and small antiviral compounds activate caspase 1 through cryopyrin producted from CIAS1 gene. ${ }^{31}$ We propose here the CIAS1 is associated with hypertension through the dominant expression of transcripts, which may depend 
on the CIAS1-VNTR genotype. To prove this hypothesis, an animal model overexpressing CIAS1 in leukocytes is desirable, which may also resolve the matter of whether CIAS1 alone activates or suppresses $\mathrm{NF} \kappa \mathrm{B}$ signaling.

A recent study showed that chronic inflammation is crucial for the development of hypertension. Plasma Creactive protein has been shown to be an independent risk factor for hypertension, ${ }^{32}$ and PMNL count in peripheral blood and increased superoxide release contributes to hypertension. ${ }^{33}$ In the animal model, the activation of $\mathrm{NF}-\kappa \mathrm{B}$ is increased in numerous tissues in stroke-porne spontaneous hypertension (SHRsp) rats compared with Sprague-Dawley (SD) rats. ${ }^{34} \mathrm{NF}-\kappa \mathrm{B}$ promotes proinflammatory genes or induces apoptosis in many organs. In leukocytes, NF- $\kappa \mathrm{B}$ can induce iNOS, cell adhesion molecules, IL-6, and other cytokines and chemokines. ${ }^{35}$ The induced inflammation generates large amount of oxidative agents, and the oxdative stress activates NF- $\kappa \mathrm{B} .{ }^{36,37}$ Thus, we predict that CIAS1 might play an important role in the development of hypertension based on inflammation brought on by NF- $\kappa \mathrm{B}$ activation.

\section{Acknowledgements}

The study was supported by a grant in aid (No.13204075) from Japanese Ministry of Education, Culture, Sports, Science and Technology. We thank Oyamada T and Nagashima $K$ for technical assistance. We thank Drs Kario, Hoshide S, Sugimoto K, Ishikawa K, Sakamoto A, (Jichi Medical School), Sekiguchi T, and Hara K (Nasu minami) Ohishi $T$ (Ohishi medical clinic) for sample correction.

\section{References}

1 Hsueh WC, Mitchell BD, Schneider JL et al: QTL influencing blood pressure maps to the region of PPH1 on chromosome 2q31-34 in Old Order Amish. Circulation 2000; 101: 2810-2816.

2 Levy D, DeStefano AL, Larson MG et al: Evidence for a gene influencing blood pressure on chromosome 17. Genome scan linkage results for longitudinal blood pressure phenotypes in subjects from the Framingham Heart Study. Hypertension 2000; 36: 477-483.

3 Perola M, Kainulainen K, Pajukanta P et al: Genome-wide scan of predisposing loci for increased diastolic blood pressure in Finnish siblings. J Hypertens 2000; 18: 1579-1585.

4 Rice T, Rankinen T, Province MA et al: Genome-wide linkage analysis of systolic and diastolic blood pressure: the Quebec Family Study. Circulation 2000; 102: 1956-1963.

5 Atwood LD, Samollow PB, Hixson JE, Stern MP, MacCluer JW: Genome-wide linkage analysis of blood pressure in Mexican Americans. Genet Epidemiol 2001; 20: 373-382.

6 Angius A, Petretto E, Maestrale GB et al: A new essential hypertension susceptibility locus on chromosome $2 \mathrm{p} 24-\mathrm{p} 25$, detected by genomewide search. Am J Hum Genet 2002; 71: 893905.

7 Caulfield M, Munroe P, Pembroke J et al: Genome-wide mapping of human locus for essential hypertension. Lancet 2003; 361: $2118-2123$.

8 Rice T, Rankinen T, Chagnon YC et al: Genomewide linkage scan of resting blood pressure: HERITAGE family study. Health, risk factors, exercise training, and genetics. Hypertension 2002; 39: $1037-1043$.
9 de Lange M, Spector TD, Andrew T: Genome-wide scan for blood pressure suggests linkage to chromosome 11 , and replication of locus on 16, 17, and 22. Hypertension 2004; 44: 872-877.

10 Intengan HD, Schiffrin EL: Vascular remodeling in hypertension: roles of apoptosis, inflammation, and fibrosis. Hypertension 2001; 38: 581-587. Review.

11 Rodriguez-Iturbe B, Vaziri ND, Herrera-Acosta J, Johnson RJ: Oxidative stress, renal infiltration of immune cells, and saltsensitive hypertension: all for one and one for all. Am J Physiol Renal Physiol 2004; 286: F606-F616. Review..

12 Russo C, Olivieri O, Girelli D et al: Anti-oxidant status and lipid peroxidation in patients with essential hypertension. J Hypertens 1998; 16: 1267-1271.

13 Berry C, Brosnan MJ, Fennell J, Hamilton CA, Dominiczak AF: Oxidative stress and vascular damage in hypertension. Curr Opin Nephrol Hypertens 2001; 10: 247-255.

14 Romeo JC, Reckelhoff JF: Role of angiotensin and oxidative stress in arterial hypertension. Hypertension 1999; 34: 943-949.

15 Wilcox CS: Reactive oxygen species: roles in blood pressure and kidney function. Curr Hypertens Rep 2002; 4: 160-166. Review.

16 Moreno MU, San Jose G, Orbe J et al: Preliminary characterisation of the promoter of the human p22(phox) gene: identification of a new polymorphism associated with hypertension. FEBS Lett 2003; 542: $27-31$.

17 Sela S, Mazor R, Amsalam M, Yagil C, Yagil Y, Kristal B: Primed polymorphonuclear leukocytes, oxidative stress, and inflammation antecede hypertension in the Sabra rat. Hypertension 2004; 44: 764-769.

18 San Jose G, Moreno MU, Olivan S et al: Functional effect of the p22phox -930A/G polymorphism on p22phox expression and NADPH oxidase activity in hypertension. Hypertension 2004; 44: $163-169$.

19 Hoffman HM, Mueller JL, Broide DH, Wanderer AA, Kolodner RD: Mutation of a new gene encoding a putative pyrin-like protein causes familial cold autoinflammatory syndrome and MuckleWells syndrome. Nat Genet 2001; 29: 301-305.

20 Mao M, Fu G, Wu JS et al: Identification of genes expressed in human CD34+ hematopoietic stem/progenitor cells by expressed sequence tags and efficient full-length cDNA cloning. Proc Nat Acad Sci 1998; 95: 8175-8180.

21 Manji GA, Wang L, Geddes BJ et al: PYPAF1, a PYRIN-containing Apaf1-like protein that assembles with ASC and regulates activation of NF-kappa B. J Biol Chem 2002; 277: 11570-11575.

22 Koonin EV, Aravind L: The NACHT family - a new group of predicted NTPases implicated in apoptosis and MHC transcription activation. Trends Biochem Sci 2000; 25: 223-224.

23 Harton JA, Linhoff MW, Zhang J, Ting JP: Cutting edge: CATERPILLER: a large family of mammalian genes containing CARD, pyrin, nucleotide-binding, and leucine-rich repeat domains. J Immunol 2002; 169: 4088-4093.

24 O'Connor Jr W, Harton JA, Zhu X, Linhoff MW, Ting JP: Cutting edge: CIAS1/cryopyrin/PYPAF1/NALP3/CATERPILLER 1.1 is an inducible inflammatory mediator with NF-kappa B suppressive properties. J Immunol 2003; 171: 6329-6333.

25 Neven B, Callebaut I, Prieur AM et al: Molecular basis of the spectral expression of CIAS1 mutations associated with phagocytic cell-mediated autoinflammatory disorders CINCA/NOMID, MWS, and FCU. Blood 2004; 103: 2809-2815.

26 Albrecht M, Domingues FS, Schreiber S, Lengauer T: Structural localization of disease-associated sequence variations in the NACHT and LRR domains of PYPAF1 and NOD2. FEBS Lett 2003; 554: 520-528.

27 Ruiz-Opazo N, Lopez LV, Herrera VL: The dual AngII/AVP receptor gene N119S/C163R variant exhibits sodium-induced dysfunction and cosegregates with salt-sensitive hypertension in the Dahl salt-sensitive hypertensive rat model. Mol Med 2002; 8: 24-32.

28 Touitou I, Lesage S, McDermott $\mathrm{M}$ et al: Infevers: an evolving mutation database for auto-inflammatory syndromes. Hum Mutat 2004; 24: 194-198. 
29 Hirakawa M, Tanaka T, Hashimoto Y, Kuroda M, Takagi T, Nakamura Y: JSNP: a database of common gene variations in the Japanese population. Nucleic Acids Res 2002; 30: 158-162.

30 Bautista LE, Vera LM, Arenas IA, Gamarra G: Independent association between inflammatory markers (C-reactive protein, interleukin-6, and TNF-alpha) and essential hypertension. J Hum Hypertens 2005; 19: 149-154.

31 Mariathasan S, Weiss DS, Newton K et al: Cryopyrin activates the inflammasome in response to toxins and ATP. Nature 2006; 440: $228-232$.

32 Kanneganti TD, Ozoren N, Body-Malapel M et al: Bacterial RNA and small antiviral compounds activate caspase-1 through cryopyrin/Nalp3. Nature 2006; 440: 233-236.

33 Kristal B, Shurtz-Swirski R, Chezar J et al: Participation of peripheral polymorphonuclear leukocytes in the oxidative stress and inflammation in patients with essential hypertension. $\mathrm{Am} \mathrm{J}$ Hypertens 1998; 11: 921-928.

$34 \mathrm{Wu}$ L, Noyan Ashraf MH, Facci M et al: Dietary approach to attenuate oxidative stress, hypertension, and inflammation in the cardiovascular system. Proc Natl Acad Sci USA 2004; 101: 7094-7099.

35 Cooke CL, Davidge ST: Peroxynitrite increases iNOS through NFkappaB and decreases prostacyclin synthase in endothelial cells. Am J Physiol Cell Physiol 2002; 282: C395-C402.

36 Sanlioglu S, Williams CM, Samavati L et al: Lipopolysaccharide induces Rac1-dependent reactive oxygen species formation and coordinates tumor necrosis factor-alpha secretion through IKK regulation of NF-kappa B. J Biol Chem 2001; 276: 30188-30198.

37 Asehnoune K, Strassheim D, Mitra S, Kim JY, Abraham E: Involvement of reactive oxygen species in Toll-like receptor 4dependent activation of NF-kappa B. J Immunol 2004; 172: 25222529.

\section{Appendix}

The Study Group of Millennium Genome Project for Hypertension:

Chairman: Tetsuro Miki (Department of Geriatric Medicine, Ehime University School of Medicine, Ehime)

Michio Yasunami, Akinori Kimura (Department of Molecular Pathogenesis, Medical Research Inst., Tokyo Medical and Dental University), Akira Hata, (Chiba University Graduate School of Medicine, Chiba), Toshio Saruta
(Department of Internal Medicine, School of Medicine, Keio University), M Yokota (Department of Clinical Pathophysiology, Nagoya University, Graduate School of Medicine), Tomohiro Katsuya, Toshio Ogihara (Department of Geriatric Medicine, Osaka University Graduate School of Medicine), Hirotsugu Ueshima (Department of Health Science, Shiga University of Medical Science), Katsuhiko Kohara, Yasuharu Tabara, Jun Nakura, Tetsuro Miki (Department of Geriatric Medicine, Ehime University School of Medicine), Sumio Sugano (Human Genome Center, Institute of Medical Science, University of Tokyo), Masayoshi Soma, Tomohiro Nakayama (Second Department of Internal Medicine, and Division of Receptor Biology, Advanced Medical Research Center, Nihon University School of Medicine), Norihiro Kato (Department of Gene Diagnostics and Therapeutics, Research Institute, International Medical Center of Japan), Ichiro Kishimoto, Kazuwa Nakao (Department of Medicine and Clinical Science, Kyoto University Graduate School of Medicine), Yuhei Kawano, Kei Kamide (Division of Hypertension and Nephrology, Department of Medicine, National Cardiovascular Center), Naoharu Iwai, Toshiyuki Miyata (Research Institute, National Cardiovascular Center), Takayuki Morisaki, (Department of Bioscience, National Cardiovascular Center Research Institute),Hitonobu Tomoike (Division of Preventive Cardiology, National Cardiovascular Center), Tsutomu Yamazaki (Department of Pharmacoepidemiology, Graduate School of Medicine,Faculty of Medicine, University of Tokyo), Katsushi Tokunaga, (Graduate School of Medicine Faculty, Intemational Health, Intemational Biomedical Sciences, University of Tokyo), Satoshi Umemura, Nobuhito Hirawa (Department of Medicine II, Yokohama City University School of Medicine), Hiroyuki Mano (Division of Functional Genomics, Jichi Medical School), Sadahiko Iwamoto (Division of Human Genetics, Center for Community Medicine, Jichi Medical School). 